\title{
Neuromyelitis optica in a 9 year old girl: A case report
}

\author{
Soutrik Seth ${ }^{1}$, Tanmoy Biswas ${ }^{2}$, Rajib Das ${ }^{3}$, Atanu Roy ${ }^{4}$ \\ ${ }^{1,2}$ Resident, ${ }^{3}$ RMO Cum Clinical Tutor, ${ }^{4}$ Associate Professor, Department of Pediatrics, Burdwan Medical College and \\ Hospital, Burdwan-713104, West Bengal, India
}

\section{A B S T R A C T}

Neuromyelitis optica (NMO) is an acquired demyelinating disorder affecting the optic nerves and spinal cord either simultaneously or sequentially. Prognosis is guarded particularly for the optic neuritis. We report here a case of first attack of NMO occurring in a 9 year old girl child coming from a rural area of Burdwan.

Key words: Demyelinating disorder, Optic neuritis, Neuromyelitis optica

Access this article online

Website:

http://nepjol.info/index.php/AJMS

DOI: 10.3126/ajms.v7i5.14780

E-ISSN: 2091-0576

P-ISSN: 2467-9100

\section{INTRODUCTION}

Neuromyelitis Optica is a rare aggressive demyelinating disorder of central nervous system characterised by recurrent attacks of optic neuritis and transverse myelitis, first reported back in 1870's. NMO was initially thought to be opticospinal form of Multiple sclerosis (MS) but with detection of the NMO antibodies (IgG antibodies directed against Aquaporin- 4 channels) it is now considered a separate disease entity. ${ }^{1-5}$ We report here a case of first attack of NMO occurring in a 9 year old girl child coming from a rural area of Burdwan.

\section{CASE REPORT}

A 9 year old girl born out of Non consangiuous marriage, resident of Galsi, Burdwan coming from a poor socio economic background was admitted in Pediatric Department of Burdwan Medical College and Hospital with the complaint of diminished vision in both eyes (right $>$ left) for last 45 days and sudden onset weakness of both lower and upper limbs (lower $>$ upper) with inability to walk and sit for last 15 days (Figure 1). There was no history of fever or trauma or convulsion or unconsciousness. Sensory function was normal. Bladder bowel function was normal.
Neurological examination revealed normal higher function status without cranial nerve involvement, hypertonia of both upper and lower limbs with power 1/5 in lower limbs and 3/5 in upper limbs, bilateral ankle clonus, brisk deep tendon reflexes. Visual aquity was diminished to finger counting (Right>left). Other systemic examinations were unremarkable.

CSF study revealed 55 cells with neutrophilic predominance, no oligoclonal bands found. Anti NMO antibodies could not be done due to financial restraints. Other blood investigations were unremarkable. MRI of whole spine revealed expansion with gross disorganisation of cervical cord at C2-C3 and C7-D6 level with multisepted cystic areas in skip fashion involving posterior aspect of cord suggestive of acute demyelination and its sequelae (Figure 2).

MRI Brain was normal study with no abnormal intensity anywhere in brain parenchyma (Figure 3). Fundoscopic evaluation showed bilateral optic neuritis. Visual evoked potential study showed axonal type both sided retino-optic pathway dysfunction(right>left) (Figure 4).

The child was treated with Methyl Prednisolone for 5 days followed by oral prednisolone for 6 weeks. Human Intravenous Immunoglobulin (IVIG) was also given for 5 days. Child improved within a week and was able to sit 
without support and stand with support (Figure 5). Child was discharged after 3 weeks with proper physiotherapy and asked to follow up regularly in OPD. In subsequent follow up there was improvement in power and gait but no improvement in vision.

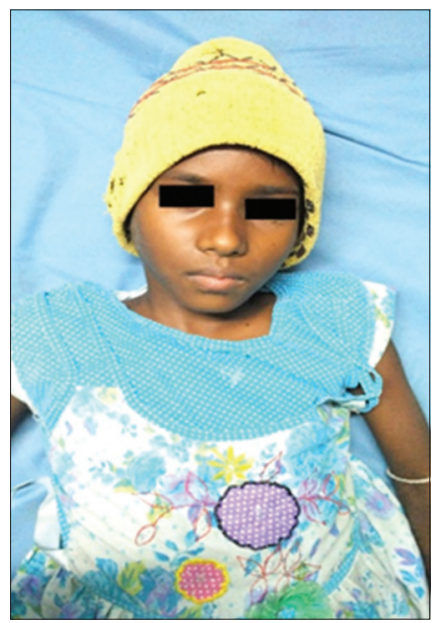

Figure 1: Child on admission

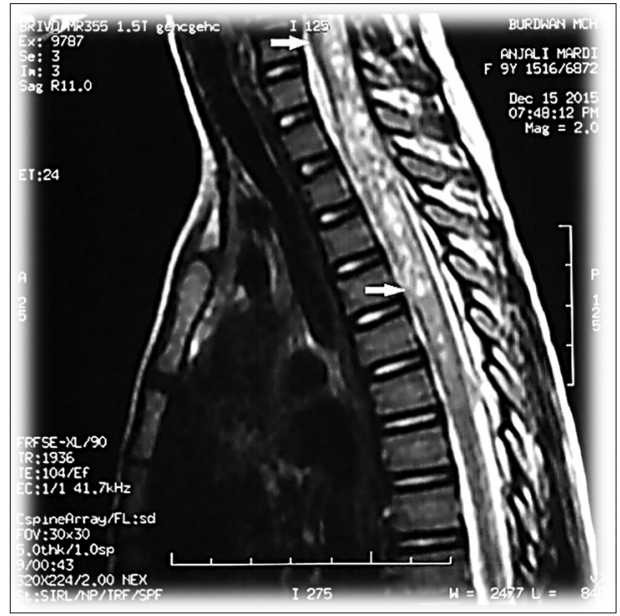

Figure 2: MRI spine showing involvement of C7-D6

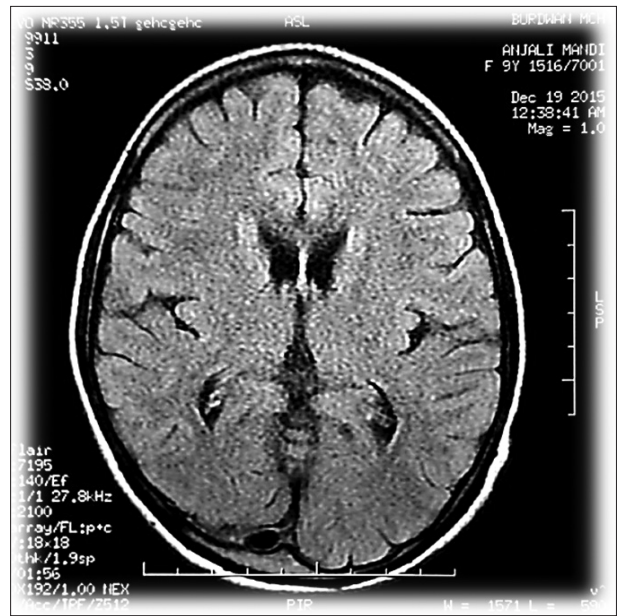

Figure 3: MRI brain within normal limits

\section{DISCUSSION}

Devic's disease or Neuromyelitis optica as the name suggests is an aggressive demyelinating disorder of the spinal cord and optic nerve affecting most commonly Asian females (3 times more common than males) in the age group $31.2 \pm 11$ years. ${ }^{1,2}$ First reported in 1870 by Sir Thomas Clifford Allbutt, later in 1894 Eugene Devic with his student Fernand Gault studied 16 patients who lost vision in one/both eyes within weeks of sudden onset weakness of limbs, recognised as optic neuritis and myelitis respectively. ${ }^{5,6}$ In 2004 Mayo Clinic researchers described a specific autoantibody against aquaporin-4 the so called NMO antibody which led to development of this new entity of $\mathrm{NMO}$ which is different from multiple sclerosis unlike the understanding before. Currently the best biomarker is Anti Myelin Oligodendrocyte glycoprotein (Anti-MOG Ab). ${ }^{6}$

NMO is usually characterized by bilateral optic neuritis (rare in MS) or unilateral, myelitis being transverse and

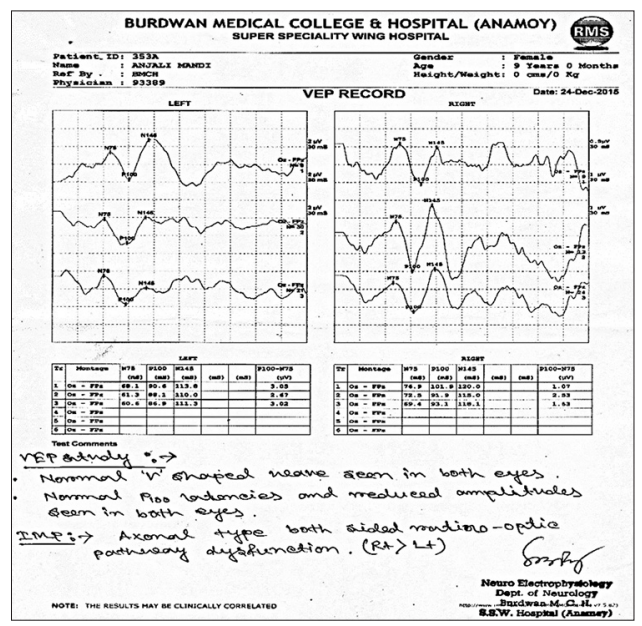

Figure 4: VEP study showing bilateral axonal type retino-optic pathway dysfunction

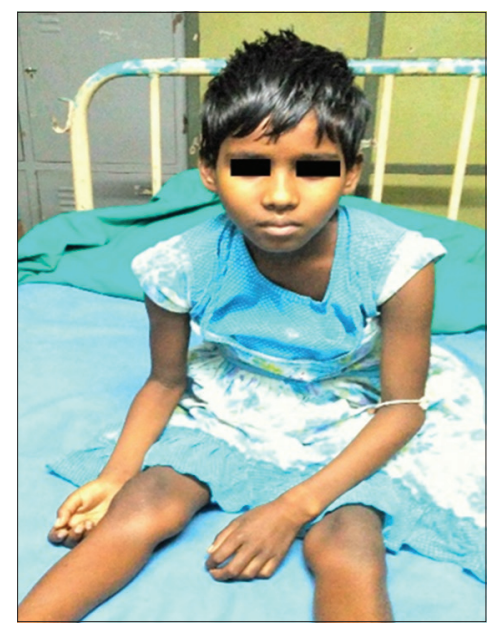

Figure 5: Child at time of discharge 
longitudinally extensive (rare in MS), progressive symptoms rare unlike MS. ${ }^{2}$

\section{DIAGNOSTIC CRITERIA FOR NEUROMYELITIS OPTICA $^{2}$}

Required:

1. Optic neuritis

2. Acute Transverse Myelitis.

Supportive (2 out of 3 criteria required)

1. Longitudinally extensive cord lesion extending over 3 or more vertebral segments

2. Brain magnetic resonance imaging normal or not meeting criteria for multiple sclerosis

3. Aquaporin-4 seropositivity.

Source: Adapted from DM Wingerchuk et al: Neurology 66:14855, 2006.

In our case 2 out of 3 supportive criteria was present.

NMO is often associated with other autoimmune disorder like SLE, Myesthenia Gravis, Sjogren Syndrome, Multiple connective tissue disorder or post infectious like EBV, CMV but no such association was found in our case. The IgG Ab against Aquaporin-4 leads to myelin destruction leading to astrocyte death and necrosis with cavitation in affected tissue. ${ }^{1,2,5}$

CSF study normally shows $>50$ white blood cells with neutrophilic predominance and increased IgG levels without oligoclonal bands. Neuroimaging shows characteristic demyelinating lesion in at least 3 spinal segments (as was in our case). Brain Neuroimaging may be normal initially (as in our case) or may show involvements not meeting criteria for MS. Treatment consists of Methylprednisolone (20-30 mg/ $\mathrm{kg} /$ day; $\max 1 \mathrm{gm} /$ day) for 3-5 days followed by prednisolone taper for 6 weeks. IVIG is also used (as in our case). Other treatment options include Plasma exchange, ${ }^{7}$ anti CD20 Ab Rituximab and Azathioprine. Rilling et al (1999) showed use of Methylprednisolone improved symptoms for a while and cyclophosphamide was used to prevent recurrence. ${ }^{3}$ Mandler et al. (1998) found no recurrence for 18 months in patients treated with prednisolone and azathioprine for 2 months. ${ }^{3,8}$

Prognosis is guarded for NMO or NMO spectrum disorders. Studies show $20 \%$ patients to be functionally blind in at least 1 eye and 31\% developed permanent monoplegia/paraplegia. $5 \mathrm{yr}$ survival in patients with paraplegia was approximately $90 \% .{ }^{1}$

\section{CONCLUSION}

Awareness to be created among paediatricians and neurologists about this rare demyelinating disorder with a generally poor prognosis so that early detection and management is possible to limit the disabilities. Also this case and similar cases which are first episodes occurring at early age needs to be regularly followed up in OPD for better assessment and prognosis.

\section{REFERENCES}

1. Jayne M. Ness (Chapter 600.2) Neuromyelitis Optica; Nelson textbook of Pediatrics $20^{\text {th }}$ ed Elsevier, 2015, p2922-2924.

2. Hauser SL and Goodin DS. (Chapter 458) Multiple Sclerosis and other demyelinating diseases; Harrison Principles of Internal Medicine $19^{\text {th }}$ ed, McGrawHill Education, 2015, p2672.

3. Silva AR, Barros SVT, Rotta NT, Ohlweiler L, Stone I and Mello LR. Journal de Pediatria; Devic's disease A case report; 0021-7557/01/77-06/522.

4. Dembinski K, Gieron-Korthals M, Martinez CR and Rodriguez L. Case report: Neuromyelitis Optica in Child: Diagnostic and Therapeutic Challenges; Volume 2013, Article ID 124929, Hindawi publishing corporation.

5. Pearce JMS. Neuromyelitis optica. Spinal Cord 2005; 43(11): 631-634.

6. Neuromyelitis optica, from Wikipedia The Free Encyclopedia [online] [cited 2016 April 19] Available from URL: https:// en.wikipedia.org/wiki/Neuromyelitis_optica.

7. M. Bonnan and Cabre P. Plasma exchange in severe attacks of neuromyelitis optica. Multiple Sclerosis International Vol. 2012, Article ID 787630, 9 pages, 2012, Hindawi publishing corporation.

8. Mandler RN, Ahmed W and Dencoff JE. Devic's neuromyelitis optica: a prospective study of seven patients treated with prednisone and azathioprine. Neurology 1998; 51(4):1219-1220. 